\title{
Situação atual na transformação entre sistemas de referência celeste e terrestre
}

\author{
Current status in the transformation between celestial and terrestrial reference systems
}

\author{
Vinicius Francisco Rofatto ${ }^{1,3}$ \\ Fabricio dos Santos Prol ${ }^{2}$ \\ Marcelo Tomio Matsuoka ${ }^{1,3}$ \\ Sérgio Florêncio de Souza ${ }^{3}$
}
${ }^{1}$ Instituto de Geografia, Curso de Engenharia de Agrimensura e Cartográfica, Universidade Federal de Uberlândia - UFU. LMG 746 km 1, s/n, Campus Monte Carmelo. Cep 38500-000. Monte Carmelo, MG, Brasil. vinicius.rofatto@ufu.br, tomiomatsuoka@gmail.com
${ }^{2}$ Programa de Pós-Graduação em Ciências Cartográficas, Universidade Estadual Paulista Júlio de Mesquita Filho - UNESP. Rua Roberto Simonsen, 305, Caixa Postal 468. Cep 19060-900.
Presidente Prudente, SP, Brasil. fabricioprol@hotmail.com
${ }^{3}$ Programa de Pós-Graduação em Sensoriamento Remoto, Universidade Federal do Rio Grande do Sul - UFRGS. Avenida Bento Gonçalves, 9500, Caixa Postal 15001. Cep 91501-970.
Porto Alegre, RS, Brasil

Recebido em 11/03/2016 - Aceito em 09/12/2016

Received on 11/03/2016 - Accepted on 09/12/2016

\begin{abstract}
RESUMO -Muitas aplicações de Geodésia, geodinâmica, Astronomia e navegação dependem da disponibilidade de referenciais geodésicos adequados. Atualmente, dois sistemas de referências são adotados por convenção: o referencial terrestre e o referencial celeste. O primeiro trata de um referencial dinâmico com eixos coordenados fixos à Terra, enquanto o segundo é um sistema inercial com eixos fixos no espaço sideral. Ambos os sistemas de referências e os procedimentos intrínsecos à eles estão baseados nas resoluções recomendadas e aprovadas pela Assembleia Geral da União Astronômica Internacional - IAU (International Astronomical Union). Uma revisão teórica é apresentada neste trabalho sobre a situação atual das resoluções do IAU. Ademais, experimentos são realizados a fim de mostrar os procedimentos necessários na transformação entre os referenciais celeste e terrestre, bem como uma aplicação é mostrada a fim de apresentar a magnitude dos erros obtidos na transformação.
\end{abstract}

Palavras-chave: Referenciais, ITRS, ICRS, Precessão, Nutação, Rotação da Terra, Movimento do Polo.

ABSTRACT - Many applications in Geodesy, geodynamics, Astronomy and navigation depend on the availability of consistent reference systems. Two conventional reference systems are adopted today for these purposes: terrestrial reference system and a celestial reference system. The first consists in a spatial reference system co-rotating with the Earth, whereas the second is an inertial system. Both reference systems and procedures are based on the International Astronomical Union resolutions. A revision of the theoretical concepts about the current state of the IAU resolutions is introduced. Moreover, experiments are performed to show the necessary procedures in the transformation between the terrestrial and celestial systems and in order to present the magnitude of the errors in the transformation.

Keywords: Reference Frames, ITRS, ICRS, Precession, Nutation, Earth's rotation, Pole Motion.

\section{INTRODUÇÃO}

O advendo da Era Espacial, marcada pelo lançamento do primeiro satélite artificial em 1957, aliado ao desenvolvimento de relógios atômicos, revolucionou a Geodésia, permitindo estudar certos fenômenos em escala global de maneira acurada. Pode-se citar o movimento de rotação da Terra, o deslocamento das placas litosféricas, os efeitos de cargas dos oceanos sobre o continente, os movimento dos satélites (artificiais e naturais) e planetas, o estudo do campo gravitacional e o monitoramento de estruturas de engenharia. A observação e a quantificação destes fenômenos requer o estabelecimento de referenciais adequados, precisos e consistentes (BLITZKOW et al., 2011; MONICO, 2008; BOCK, 1996). Dentro deste contexto, dois referenciais têm sido adotados por convenção e utilizados para as diversas aplicações envolvidas na Astronomia, Engenharia e Geociências: o referencial terrestre e o referencial celeste.

O Sistema de Referência Celeste (CRS - Celestial Reference System) é definido com a origem e os eixos cartesianos fixos em pontos no espaço sideral, permitindo descrever o movimento das galáxias, das estrelas, do Sol, dos planetas, dos satélites, dentre outros. 
Uma vez que os objetos de interesse são observados sobre a superfície terrestre, a complexidade dos sistemas de referência é menor quando os pontos que definem os objetos são referenciados em um Sistema de Referência Terrestre (TRS - Terrestrial Reference System). O TRS acompanha a Terra em seu movimento de rotação e de translação ao redor do Sol e tem sua origem no centro de massa da Terra, ou num conceito atual, no geocentro (PLAG et al., 2009; BLITZKOW et al., 2011).

Atualmente, um grande número de observações sobre a Terra é feito por meio de sensores espaciais. Apesar das observações de pontos localizados na superfície terrestre serem referenciadas ao TRS, a órbita dos sensores espaciais está vinculada a corpos no espaço sideral, que se movimentam ao redor do centro de massa da Terra. Desta forma, a órbita destes sensores é mais bem descrita quando os mesmos são referenciados ao CRS. Em algumas aplicações, é necessário realizar a transformação entre os referenciais CRS e TRS, e viceversa. Por exemplo, no posicionamento pelos Sistema Global de Navegação por Satélites (GNSS - Global Navigation Satellite System), as posições dos satélites são descritas no sistema CRS, ao passo que as estações terrestres são representadas em um sistema fixo à Terra e rotacionam com ela, ou seja, no TRS. Neste caso, para que as posições dos satélites e as estações terrestres sejam representadas no mesmo sistema de referência, é necessário conhecer a relação matemática entre os referenciais (MONICO, 2008). Os parâmetros que relacionam estes dois sistemas são as coordenadas do polo e os parâmetros de orientação da Terra, os quais são calculados diariamente pelos serviços internacionais de Geodésia e Astronomia.

A Associação Internacional de Geodésia IAG (International Association of Geodesy), a União Astronômica Internacional IAU (International Astronomical Union), e a União Geodésica e Geofísica Internacional IUGG (International Union of Geodesy and Geophysics, têm empenhado esforços na definição e realização dos sistemas referências, bem como na determinação dos parâmetros que relacionam os sistemas. Neste sentido em 1987, a IAU e a IUGG criaram um serviço denominado International Earth Rotation Service, renomeado em 2003 para International Earth Rotation and Reference Systems Service, mantida até hoje com a sigla IERS. O IERS, que teve seu funcionamento no dia $1^{\circ}$ de Janeiro de 1988, substituindo o IPMS (International Polar Motion Service) e a sessão de rotação da Terra do BIH (Bureau International de l'Heure), é responsável, entre outras funções, pela:

- definição, materialização e a manutenção de um sistema de referência terrestre, o qual é baseado em técnicas geodésicas espaciais de observações de alta precisão;

- definição, materialização e manutenção de um sistema de referência celeste baseado em observações de objetos extragaláctios via tecnologia VLBI (Very Long Baseline Interferometry); e

- determinação dos parâmetros de orientação da Terra (EOP - Earth Orientation Parameters), que servem para vincular os dois sistemas, em conjunto com modelos de precessão e nutação (IERS, 2010).

Atribui-se ao IERS, nortear os sistemas de referências modernos e os procedimentos inerentes a eles. Os modelos, as constantes e os padrões dos sistemas de referência são constantemente atualizados e definidos por convenções, sendo a edição mais atual denominada de IERS Conventions 2010, a qual é baseada em uma série de resoluções aprovadas e recomendadas pelas uniões científicas internacionais. Assim, os referenciais, quer celeste, quer terrestre, são adotados por convenção, dando origem ao sistema de referência convencional.

O sistema celeste é baseado na Resolução A4 ${ }^{1}$, aprovada na Assembleia Geral da IAU em 1991. O sistema foi oficialmente iniciado pela Resolução B2 da IAU em 1997, sob a denominação de Sistema de Referência Celeste Internacional (ICRS - International Celestial Reference System) e sua definição foi refinada pela Resolução B1 da IAU em 2000 e pela Resolução B3 da IAU em 2009. Já o sistema terrestre é baseado na Resolução 2 da IUGG em 1991, e foi formalmente apresentado como Sistema de Referência Terrestre Internacional (ITRS - International Terrestrial Reference System) pela Resolução 2, aprovada na XXIV Assembleia Geral da IUGG em 2007. A transformação entre os referenciais terrestre e celeste é baseada na Resolução B1 da IAU de 2000 e foi complementada pelas Resoluções B1 e B2 da IAU em 2006. (MONICO, 2004; CAPITAINE, 2009; IERS, 2010; ALTAMIMI e COLLILIEUX, 2013). Detalhes sobre as resoluções podem ser encontrados na seguinte página web: https://www.iau.org/administration/resolutions/general_as semblies/.

No posicionamento por satélite, os referenciais de interesse são geocêntricos, pois os satélites têm como origem de seu movimento o centro de massa da Terra. Logo, em termos de referenciais celeste e terrestre, o interesse é pelo GCRS (Geocentric Celestial Reference System - Sistema de Referência Geocêntrico) e o ITRS, respectivamente. Classicamente, a transformação do GCRS para o ITRS é efetuada usando uma sequência de rotações que levam em consideração a precessão, a nutação, a rotação e orientação da Terra, incluindo o movimento do polo. Com a introdução da resolução B1 da IAU 2006 um novo modelo ${ }^{2}$ de precessão foi adotado oficialmente para complementar o de nutação da IAU 2000A.

Desta forma, o presente trabalho apresenta os conceitos básicos envolvidos nos referenciais terrestres e celestes, bem como uma breve evolução histórica destes referenciais. Visto a constante evolução dos referenciais, objetiva-se também apresentar os procedimentos para realizar a transformação entre referenciais celeste e terrestre por meio de exemplos práticos, considerando as

${ }^{1}$ Introduziu explicitamente a Teoria da Relatividade Geral, como base para a definição e realização de referenciais (McCARTHY, 1992).

${ }^{2}$ Conhecido como Teoria da precessão P03 de Capitaine et al., 2003) ou modelo de precessão IAU 2006. 
resoluções em vigor. Neste sentido, as seções 2 e 3 apresentam uma revisão sobre a definição dos sistemas celeste e terrestre, respectivamente; a seção 4 mostra os conceitos utilizados na transformação entre tais sistemas e a seção 5 mostra resultados experimentais obtidos ao realizar a transformação destes referenciais ao considerar a órbita dos satélites do GNSS.

\section{REFERENCIAL CELESTE}

A origem e os eixos de um referencial celeste não podem ser materializados de forma direta. Eles são definidos por convenção e é necessário escolher uma estrutura física que sirva de base para a sua materialização. Durante os anos de 1962 a 1983 utilizouse um conjunto de 1535 estrelas do catálogo FK4, cujas coordenadas uranográficas, ascensão reta e declinação, eram conhecidas. A partir de 1983, o Astronomisches Rechen Institut compilou um novo catálogo, o FK5, que aperfeiçoou a descrição dos movimentos individuais das estrelas e o sistema usou os valores convencionais de precessão descritos pelo IAU 1976 (SEEBER, 2003).

Em 1991, nas recomendações sobre Sistemas de Referência, a IAU optou por objetos extragalácticos distantes, em substituição às estrelas, que definissem de forma mais acurada um referencial celeste, e adotou direções que fossem fixas em relação a um conjunto selecionado desses objetos. De conformidade com esta recomendação, o ICRS passou a ser materializado pelo ICRF através de um catálogo de coordenadas equatoriais (uranográficas), sendo elas a ascensão reta e a declinação de objetos extragalácticos, conhecidos como Quasars (Quasi Stelar Radio Source). Estas coordenadas uranográficas são obtidas a partir de observações VLBI (Very Long Baseline Interferometry), relativas ao equador médio em J2000, e permitem estimar correções aos modelos de precessão e nutação do IAU (BLITZKOW et. al., 2011).

Uma vantagem fundamental dos Quasars é que suas distâncias possuem magnitudes tão grandes ( 10 bilhões de anos-luz), que seus movimentos próprios são negligenciáveis em comparação com a precisão atual das observações VLBI, o que garante uma estabilidade rotacional ao referencial. Mesmo a paralaxe oriunda de uma suposta observação a partir do centro de massa da Terra ou do baricentro do sistema solar é desprezível.

A primeira realização do ICRS, conhecido como ICRF1, foi feita em 1995 utilizando as posições de 608 quasars. Atualmente, tem-se o ICRF-2, segunda realização do ICRF, a qual foi adotada oficialmente por meio da Resolução B3 da XXVII Assembleia da IAU em 2009. Ela é composta por 3414 fontes de rádio extragalácticos com estabilidade ao nível de $10 \mu$ as (microarco de segundos) - algo em torno de 2 vezes mais estável que o ICRF1. A Fig. 1 mostra a distribuição dos quasars na realização do ICRF-2.

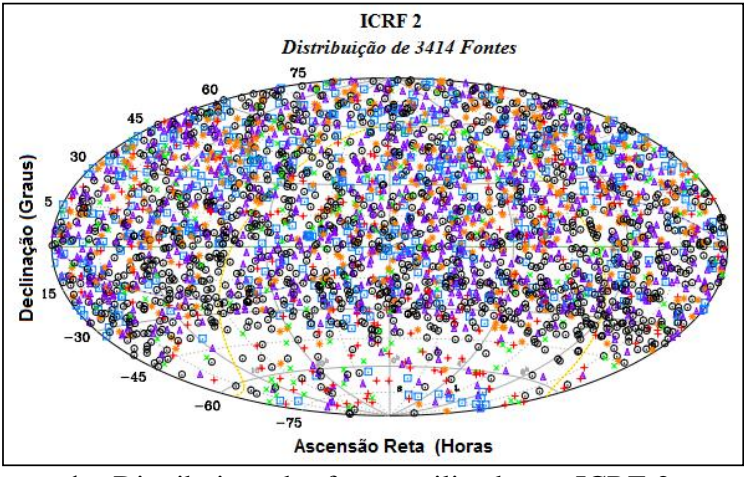

Figura 1 - Distribuição das fontes utilizadas no ICRF-2.

Fonte: Adaptado de Ma et al., (2009).

Uma nova realização, denominado ICRF-3, está sendo discutida com a finalidade de refinar o sistema celeste por meio da redução do vazio de observações na região sul do planeta devido à ausência de estações VLBI e da redução de erros sistemáticos encontrados nas observações VLBI com bandas de frequência $\mathrm{S}$ e $\mathrm{X}$ (frequência de 2,3 e 8,4 GHz e comprimentos de onda de $13 \mathrm{~cm}$ e $3,6 \mathrm{~cm}$, respectivamente), dentre outros refinamentos (MALKIN et al., 2014).

Dentro do conceito da relatividade geral, a origem do ICRS é o baricentro do sistema solar, produzindo o Sistema de Referencia Celeste Baricêntrico (BCRS Barycentric Celestial Reference System) e, com isso ficou definido, por convenção, o ICRS do IERS, tendo as seguintes características (BLITZKOW et. al., 2011)

- Origem no baricentro do sistema solar;

- Eixo X orientado segundo o equinócio vernal do equador médio na época J2000;

- Eixo $\mathrm{Z}$ orientado para o polo de referência convencional na época $\mathbf{J} 2000$;

- Eixo Y completa o sistema dextrógiro.

As características do ICRS permitem assegurar que, para todos os efeitos, o ICRS é um sistema inercial (BLITZKOW et. al., 2011). No entanto, em algumas aplicações, faz-se uso do GCRS, onde a origem do ICRS é no geocentro (MONICO, 2008). Para aplicações em Geodésia, poderia ser utilizado apenas o ICRS, porém, a complexidade dos sistemas de referência é menor quando os pontos estão referenciados em um sistema terrestre, com eixos cartesianos fixos a Terra e que se movimentam juntamente com a mesma.

\section{REFERENCIAL TERRESTRE}

O ITRS é tradicionalmente adotado como sistema de referência terrestre internacional, no qual se concretiza por meio do ITRF (International Terrestrial Reference Frame). Historicamente, o ITRF teve início em 1984, com a combinação do TRF, nomeada BTS84. Neste, utilizaram-se coordenadas de estações derivadas das técnicas VLBI, LLR (Lunar Laser Ranging), SLR 
(Satellite Laser Ranging) e DOPPLER, sendo iniciado como atividade do $\mathrm{BIH}$ (Bureau International de L'Heure). O BIH era o centro de coordenação internacional do monitoramento do eixo de rotação da Terra, por meio do projeto MERIT (Monitoring of Earth Rotation and Intercomparison of Techniques). Outras três realizações do sistema terrestre do BIH (BTS - BIH Terrestrial System) foram efetuadas em 1985, 1986 e 1987. Em 1988, foi criado o IERS pela IUGG e IAU, onde se deu a primeira realização do IERS, identificada como ITRF88.

A ideia principal do ITRF é combinar as posições e velocidades de estações sobre responsabilidade de diferentes centros de análises, utilizando observações de diferentes técnicas geodésicas espaciais (VLBI, SLR/LLR, DORIS, GNSS) e disponibilizar listas de coordenadas e velocidades deste grupo de estações com as respectivas precisões (PLAG e PEARLMAN, 2009).

De acordo com a resolução $\mathrm{n}^{\circ} 2$ da IUGG, adotada em Viena em 1991, o ITRS deve atender as seguintes definições (MONICO, 2008):

- $\quad$ Origem geocêntrica, o que contempla o centro de massa da Terra, oceanos e atmosfera;

- Escala consistente com o Tempo Coordenado Geocêntrico (TCG) para um dado referencial geocêntrico;

- Orientação inicial definida pelo BIH, na época 1984,0;

- Evolução temporal da orientação assegurada pela condição NNR (No Net Rotation) em relação ao movimento tectônico horizontal sobre a Terra;

- Eixo Z aponta na direção do CTP (Conventional Terrestrial Pole);

- Eixo X é orientado na direção média do meridiano de Greenwich;

- $\quad$ Eixo Y torna o sistema dextrógiro.

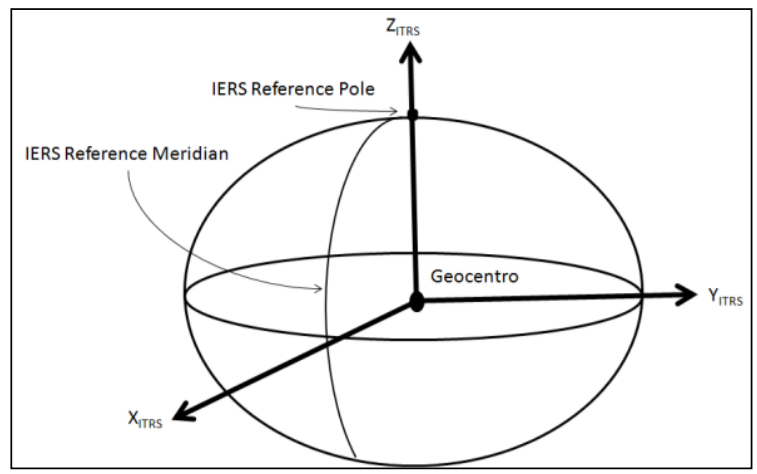

Figura 2 - Representação do ITRS.

Fonte: Autor.

A origem do ITRS é determinada por tecnologias espaciais, tais como LLR e SLR, que estão vinculadas a corpos que se movimentam ao redor do centro de massa da Terra. Na verdade, as observações oriundas das referidas tecnologias têm suas limitações e imperfeições e há questionamentos sobre as redistribuições de massa na Terra, o que levanta dúvidas sobre a real posição do centro de massa da Terra, obtida a partir das observações espaciais. Isto levou a considerar a ideia de que a origem do referencial pode não ter uma perfeita consistência com o centro de massa e, por isso, é denominada de geocentro (BLITZKOW et. al., 2011).

A escala depende de algum parâmetro físico como, por exemplo, a constante gravitacional geocêntrica (GM) ou a velocidade da luz (c) (BLITZKOW et. al., 2011). O VLBI é excelente para proporcionar a escala do referencial, porém outras técnicas, como o GNSS e DORIS (Doppler Orbitography and Radio Positioning Integrated by Satellite), também auxiliam na sua realização. Estas técnicas são inter-ligadas via conexões locais na inter-solução final (MONICO, 2006), sendo que os métodos empregados para a amarração das soluções (métodos clássicos de levantamento geodésico ou com uso do GNSS), não acompanham a precisão das técnicas espaciais. Isto acarreta em algumas deficiências nas soluções finais do ITRF (ALTAMIMI et al., 2011).

A orientação, que não pode ser observada por nenhuma das técnicas (VLBI, LLR/SLR, GNSS, DORIS), foi estabelecida convencionalmente e não é possível determinar qualquer variação da mesma com o tempo. Assim, recomendou-se definir a evolução da orientação no tempo através da chamada condição de não rotação da rede (NNR - No Net Rotation) com respeito a movimentos horizontais tectônicos sobre toda a superfície da Terra. Esta condição fica responsável, então, por alinhar a orientação da rede aos modelos geológicos que descrevem as rotações das placas litosféricas, como o modelo NNRNuvel-1A e o APKIM (BLITZKOW et. al., 2011).

As evoluções nas técnicas geodésicas espaciais de observação e o aprimoramento de modelos matemáticos, faz com que os sistemas de referência sejam constantemente aperfeiçoados. Hoje, tem-se o ITRF2014, a nova realização do ITRS. Ele apresenta duas principais características que não constam nas versões anteriores, são elas:

- Estimativas das oscilações anual e semi-anual a partir de análises de séries temporais;

- Modelo de Deformação Pós-Sísmica (PSD - PostSeismic Deformation) para aqueles usuários que desejam computar a posição de uma determinada estação terrestre afetada por deformações póssísmica em uma determinada época.

Os leitores poderão encontrar mais detalhes na página web: http://itrf.ign.fr/ITRF_solutions/2014/.

\section{TRANSFORMAÇÃO ENTRE REFERENCIAIS CELESTE E TERRESTRE}

A transformação entre os sistemas celeste e terrestre é baseada na resolução B1 da IAU (2000) e foi complementada pelas resoluções B1 e B2 da IAU (2006). Com a introdução da resolução B1 da IAU de 2000, os modelos de precessão e nutação até então adotados (IAU 1976 e IAU 1980) foram substituídos pelo IAU2000A, 
que proporciona precisão na ordem de 0,2 mas (miliarco de segundos), e IAU 2000B com precisão inferior em relação à IAU2000A, em torno de 1,0 mas (MATHEWS et al., 2002; McCARTHY e LUZUM, 2002).

O modelo de precessão da IAU2000A corrige apenas as variações de precessão fornecida pelo modelo IAU76 e, portanto, não corresponde a uma teoria dinâmica. Em vista disso, a Resolução IAU B1 2006, proposta pelo Grupo de Trabalho da seção de Precessão e Eclíptica, recomendou que, no início do dia 1 de Janeiro de 2009, as componentes do modelo de precessão da IAU2000A seja substituída pela teoria da precessão P03 de Capitaine et al. (2003), a fim de ser consistente tanto com a teoria dinâmica como o modelo de nutação IAU 2000A. Este modelo é referido na literatura como precessão IAU 2006. A resolução B2 da IAU 2006, a qual é suplementar as resoluções da IAU 2000 para sistemas de referência, redefiniu a origem do equador, substituindo o CEO (Celestial Ephemeris Origin) e TEO (Terrestrial Ephemeris Origin) para CIO (Celestial Intermediate Origin) e TIO (Terrestrial Intermediate Origin), respectivamente (CAPITAINE, 2012). Além disso, inseriu a definição do sistema de referência celeste e terrestre intermediário, sob a sigla CIRS (Celestial Intermediate Reference System) e TIRS (Terrestrial Intermediate Reference System), respectivamente. Baseado também nas resoluções da IAU 2000, o polo celeste de observação ficou definido como sendo o CIP (Celestial Intermediate Pole) - ver Fig. 3. A Fig. 4 mostra alguns elementos principais na transformação dos sistemas de referência.

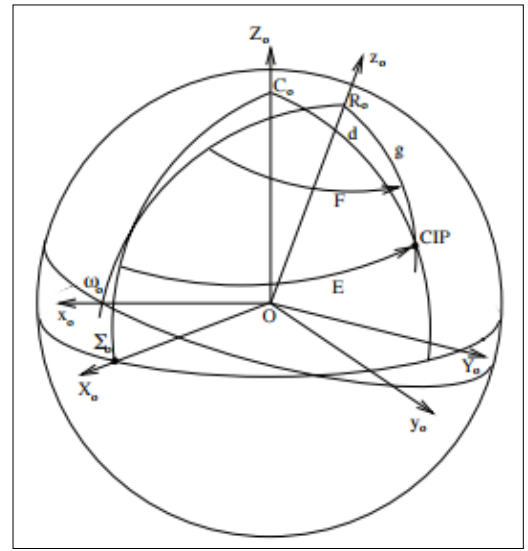

Figura 3 - Orientação do CIP

Fonte: Adaptado de Capitaine e Soffel, (2015).

Na Fig. 3, E e d são as coordenadas do CIP no GCRS ( $\operatorname{com} X=\operatorname{sen}(d) \cos (E) ; Y=\operatorname{sen}(d) \operatorname{sen}(E)) ; F$ e $g$ são as coordenadas do CIP no sistema ITRS ( $\mathrm{x}=$ $\operatorname{sen}(\mathrm{g}) \cos (\mathrm{F}) ; \quad \mathrm{y}=-\operatorname{sen}(\mathrm{g}) \operatorname{sen}(\mathrm{F})$. O polo do GCRS é denotado por $\mathrm{Co}$, e a origem do equador no GCRS é denotado por $\Sigma$ o.

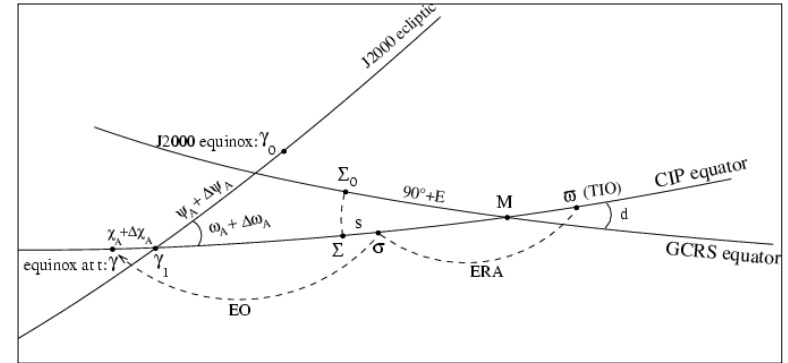

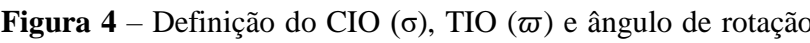
da Terra (ERA).

Fonte: Adaptado de Capitaine e Soffel, (2015).

Na Fig. 4, ERA é o ângulo de rotação da Terra ao longo do equador do CIP; $E O$ é a equação das origens que relaciona o $\mathrm{CIO}(\sigma)$ com o equinócio $\gamma$ em um determinado instante $t ; \gamma_{0}$ e $\gamma_{1}$ são as intersecções da eclíptica em J2000 com o equador do GCRS em J2000 e equador do CIP, respectivamente. As quantidades $\psi_{A}+$ $\Delta \psi_{A}$ e $\varpi_{A}+\Delta \varpi_{A}$ são a precessão e a nutação em longitude e obliquidade referentes à eclíptica na época J2000; e $\chi_{A}+\Delta \chi_{A}$ é o movimento da eclíptica no equador do CIP na época J2000. O CIRS é definido pelo CIP e CIO, e o TIRS pelo equador intermediário do CIP e o TIO.

Desta forma, nota-se que a transformação entre o referencial celeste, GCRS, e referencial terrestre, ITRS, depende dos movimentos da Terra, que podem ser representados pelos parâmetros dependentes da orientação da Terra $(E O P)$, precessão, nutação, movimento polar e o ângulo de rotação.

O CIP não possui significado físico, mas representa um polo intermediário entre o GCRS e o ITRS. Para tanto, é necessária a utilização de offsets, diferentes para cada época, nos modelos convencionais da precessão e nutação e na orientação da Terra. Com isso, o CIP possui um offset em relação ao polo do GCRS, para assim manter consistência com os modelos da IAU. Tal movimento do polo é calculado pelo IERS por meio de observações astro-geodésicas, no qual são monitoradas as variações do CIP no GCRS, denominadas de "celestial pole offset" (IERS, 2010).

Dado os parâmetros do CIP em um dado instante $(\mathrm{t})$, a transformação das coordenadas celestes $\bar{X}^{C}$ em terrestres $\bar{X}^{T}$ é obtida por:

$$
\bar{X}^{T}=W(t) R(t) Q(t) \bar{X}^{C}
$$

Na Eq. 1 tem-se $W$ a matriz de rotação resultante do movimento do polo, $R$ a matriz de rotação devido ao ângulo de rotação da Terra e $Q$ a matriz de rotação resultante do movimento do polo celeste, no sistema celeste (precessão e nutação).

Já a inversa da Eq. 1 representa a transformação entre coordenadas terrestre em celeste, sendo expressa por:

$$
\bar{X}^{C}=Q(t) R(t) W(t) \bar{X}^{T}
$$


De acordo com as resoluções do IAU 2000, tal transformação representa a posição do CIP no GCRS corrigido da nutação e precessão $(Q)$, do movimento do polo $(W)$, e do ângulo de rotação da Terra $(R)$. Estes parâmetros são medidos no instante $t$, que pode ser obtido por meio de:

$$
t=(T T-2451545 / 3625),
$$

sendo,

$$
T T=T A I+32,184=T_{G P S}+19+32,184
$$

Na Eq. 4 TT representa o Tempo Terrestre, TAI é o Tempo Atômico e $T_{G P S}$ é o tempo GPS (Global Positioning System), ver Monico (2008) para melhor compreensão destes termos.

Vale ressaltar que a rotação de matrizes para um dado ângulo $(\beta)$ é obtida por meio de:

$$
\begin{aligned}
R_{1} & =\left[\begin{array}{ccc}
1 & 0 & 0 \\
0 & \cos \beta & -\sin \beta \\
0 & \sin \beta & \cos \beta
\end{array}\right] \\
R_{2} & =\left[\begin{array}{ccc}
\cos \beta & 0 & \sin \beta \\
0 & 1 & 0 \\
-\sin \beta & 0 & \cos \beta
\end{array}\right] \\
R_{3} & =\left[\begin{array}{ccc}
\cos \beta & -\sin \beta & 0 \\
\sin \beta & \cos \beta & 0 \\
0 & 0 & 1
\end{array}\right]
\end{aligned}
$$

\subsection{Movimento do Polo}

O movimento do polo é a rotação do polo celeste verdadeiro (eixo de rotação instantâneo) em relação ao polo de um sistema de referência convencional fixo a Terra, denominado de CTP. Como se trata de ângulos pequenos, elas podem ser vistas como coordenadas cartesianas próximas ao polo de referência (ALVES, SOUZA E MONICO, 2008).

O movimento polar afeta as coordenadas de todos os pontos terrestres. Esse movimento é causado por variações na distribuição de massa da Terra e de sua atmosfera, as quais podem ocorrer devido a fenômenos meteorológicos, geológicos e geofísicos (MONICO, 2006). Para a transformação entre referenciais terrestres e celestes, a matriz correspondente ao movimento do polo é obtida por:

$$
W(t)=R_{3}\left(-s^{\prime}\right) R_{2}\left(x_{P}\right) R_{1}\left(y_{P}\right),
$$

onde $x_{P}$ e $y_{P}$ são as coordenadas do CIP no ITRS e $s^{\prime}$ é uma quantidade que proporciona a posição do TIO no equador do CIP. A expressão do $s^{\prime}$ é dada em função das coordenadas $x_{P}$ e $y_{P}$, e suas variações no tempo $\dot{\mathrm{x}}_{\mathrm{p}}$ e $\dot{\mathrm{y}}_{\mathrm{p}}$, respectivamente:

$$
s^{\prime}=\frac{1}{2} \int_{t_{0}}^{t}\left(x_{\mathrm{p}} \dot{\mathrm{y}}_{\mathrm{p}}-y_{\mathrm{p}} \dot{\mathrm{x}}_{\mathrm{p}}\right) d t
$$

\section{2 Ângulo de Rotação da Terra}

O ângulo de rotação da Terra (ERA - Earth Rotation Angle) é definido como o ângulo, medido ao longo do equador do CIP, entre o CIO e o TIO, sendo UT1 a medida de tempo adotada. A matriz de transformação que rotaciona o CIO ao longo dos eixos do CIP é expressa por:

$$
R(t)=R_{3}(-\theta),
$$

onde $\theta$ é o ângulo de rotação da Terra (ERA), que pode ser obtido por (Capitaine et al., 2000):

$$
\begin{aligned}
\theta(T u)= & 2 \pi(0,7790572732640+ \\
& 1,002273781191135448 T u),
\end{aligned}
$$

com

$$
T u=J D(U T 1)-2451545
$$

$$
U T 1=U T C+D U T 1
$$

sendo DUT1 uma correção periódica do UTC (Tempo Universal Coordenado - Universal Time Coordinate), constantemente divulgada pelo Boletim D do IERS, e JD uma função de conversão para o dia Juliano (Julian Day). Para detalhes de cálculo dos sistemas de tempo ver Monico (2008).

\subsection{Precessão e Nutação}

Os movimentos de Precessão e Nutação são efeitos resultantes da atração gravitacional do Sol e da Lua sobre a protuberância equatorial da Terra, o que implica que o movimento total resultante possa ser decomposto em uma componente principal secular (Precessão) e em uma componente secundária periódica (Nutação) (MONICO, 2008), como visto na Fig. 5.

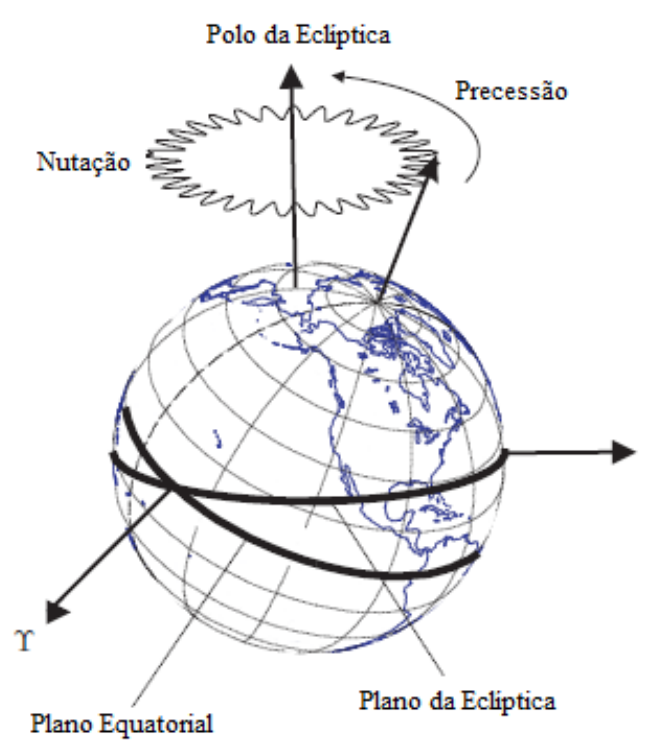

Figura 5 - Representação dos efeitos de precessão e nutação. Fonte: Adaptado de https://gmat.gsfc.nasa.gov/ 
A matriz de rotação que corrige o movimento do CIP no GCRS por meio de modelos de Nutação e Precessão do IAU é dada por (IERS, 2010):

$$
Q(t)=\left[\begin{array}{ccc}
1-a X^{2} & -a X Y & X \\
-a X Y & 1-a Y^{2} & Y \\
-X & -Y & 1-a\left(X^{2}+Y^{2}\right)
\end{array}\right] R_{3}(s),
$$

$\mathrm{Na}$ Eq. 13, s é identificado como "CIO locator", ou seja, é um parâmetro que provê a posição do CIO no equador do CIP. O parâmetro $a$ é dado por:

$$
a=\frac{1}{2}+\frac{1}{8}\left(X^{2}+Y^{2}\right)
$$

sendo que $X$ e $Y$ definem a posição do CIP no GCRS Mais detalhes da obtenção dos parâmetros de Precessão e Nutação são apresentados em Capitaine (2012); Capitaine et al., (2003) e Capitaine e Soffel, (2015).

\section{RESULTADOS E DISCUSSÃO}

Este capítulo apresenta análises quantitativas e visuais do uso das transformações entre os referencias celeste e terrestre, confrontando a mais atual resolução do IAU com sua antecessora.

As rotinas utilizadas para realizar as transformações são disponibilizadas, publicamente, pelo SOFA (Standards of Fundamental Astronomy) no seguinte endereço eletrônico: http://www.iau-sofa.rl.ac.uk. O código fonte para os devidos cálculos da resolução IAU 2000A e IAU 2000B é disponibilizado tanto em linguagem de programação $\mathrm{C} / \mathrm{C}++$ quanto em Fortran. Neste trabalho, a implementação foi realizada em $\mathrm{C} / \mathrm{C}++$, junto ao compilador GCC (GNU Compiler Collection). As principais rotinas utilizadas são apresentadas na Tab. 1.

Tabela 1 - Rotinas utilizadas na transformação entre os referenciais

$$
\text { Rotinas }
$$

\begin{tabular}{|c|c|}
\hline iauCal2jd & $\begin{array}{l}\text { Converte os dados do calendário } \\
\text { gregoriano para o Juliano }\end{array}$ \\
\hline iauDat & Calcula TAI - UTC \\
\hline iauXys00a & $\begin{array}{c}\text { Calcula as coordenadas do CIP usando } \\
\text { o modelo IAU2000A de precessão e } \\
\text { nutação }\end{array}$ \\
\hline iauXys06a & $\begin{array}{c}\text { Calcula as coordenadas do CIP usando } \\
\text { o modelo IAU2006/2000A de } \\
\text { precessão e nutação }\end{array}$ \\
\hline iauC2ixys & $\begin{array}{c}\text { Converte GCRS para o CIRS } \\
\text { (Celestial Intermediate Reference } \\
\text { System) }\end{array}$ \\
\hline iauEra00 & Ângulo de Rotação da Terra \\
\hline iauCR & Copia uma Matriz \\
\hline iauRz & Matriz de rotação $R_{3}$ \\
\hline iauPom00 & Matriz do movimento do polo \\
\hline iauRxr & Multiplicação de duas matrizes \\
\hline
\end{tabular}

Função

As coordenadas do CIP são fornecidas pelo Boletim $\mathrm{B}$ do IERS, com $\mathrm{x}_{\mathrm{p}}=0,070598$ " e $\mathrm{y}_{\mathrm{p}}=0,396865$ ", e $D U T 1=+0,1 s$ para 2 de junho de 2013 .

Com o intuito de ilustrar o uso das resoluções IAU2000A/2006 em vigor para fins de posicionamento GNSS, fez-se a transformação entre os referenciais celeste e terrestre. Para isso, as coordenadas do satélite do sistema GPS PRN 1, para o dia 2 de junho de 2013, no referencial terrestre $\mathrm{IGb}^{3} 8^{3}$, foi transformada para o sistema GCRS. A Fig. 6 mostra as coordenadas do satélite no ITRS e a Fig. 7 no GCRS.

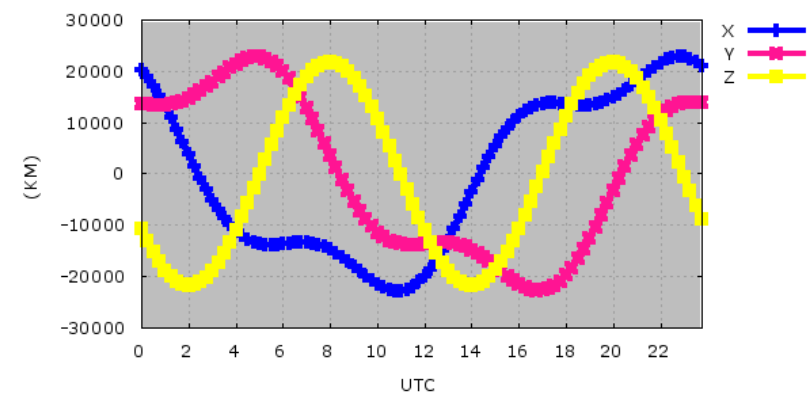

Figura 6 - Coordenadas do PRN 1 (GPS) no ITRS $(02 / 06 / 2013)$.

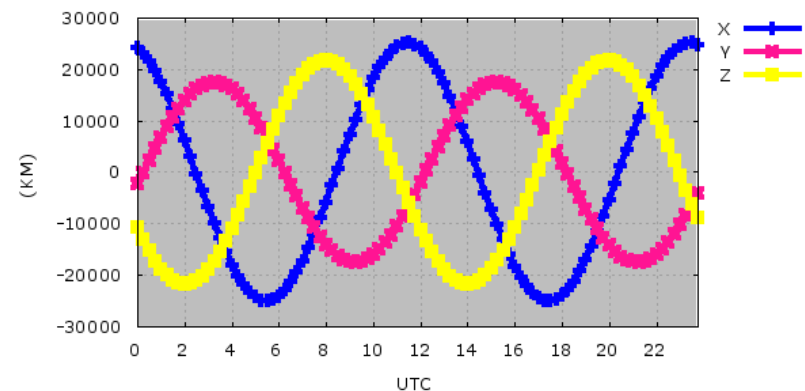

Figura 7 - Coordenadas do PRN 1 (GPS) no GCRS $(02 / 06 / 2013)$

Para uma melhor visualização da órbita do satélite, a Fig. 8 e Fig. 9 apresentam a variação das coordenadas do satélite no referencial ITRS e ICRS, respectivamente, de forma tridimensional.

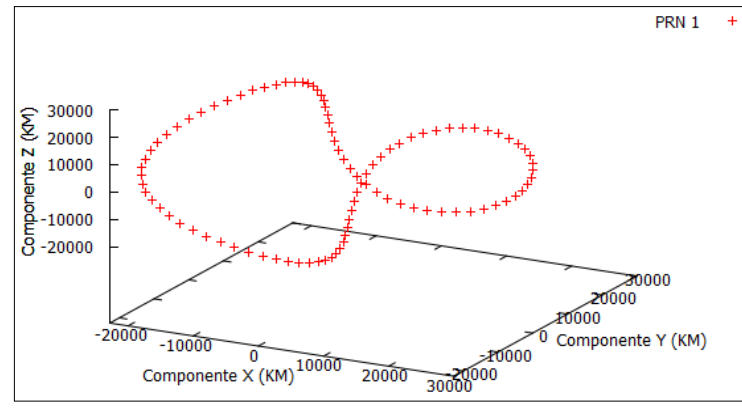

Figura 8 - Coordenadas 3D do PRN 1 (GPS) no ITRS $(02 / 06 / 2013)$.

${ }^{3}$ A realização atual do ITRS pelo IGS é denominada de IGb08. As efemérides precisas (SP3) do IGS (International GNSS Service) foram utilizadas neste trabalho para obter as coordenadas dos satélites da constelação GPS. 


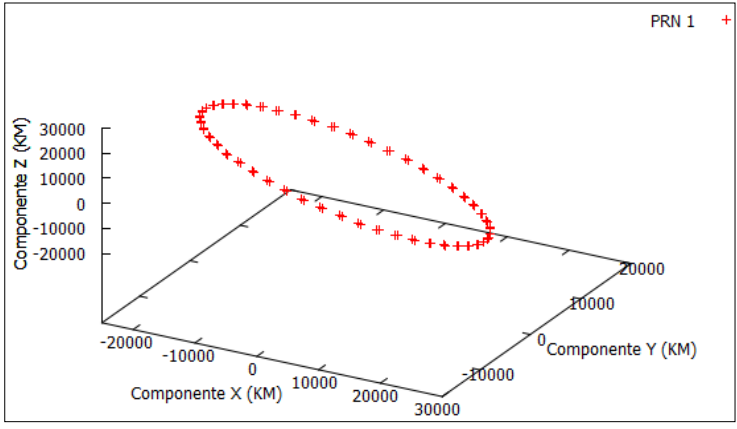

Figura 9 - Coordenadas 3D do PRN 1 (GPS) no GCRS $(02 / 06 / 2013)$.

Nota-se que pelas Fig. 6 e Fig. 7 que as coordenadas apresentam comportamentos diferentes nos diferentes referenciais. A razão disso é o movimento de rotação da Terra, pois o sistema terrestre rotaciona com ela, o que não ocorre no celeste. Desta forma, os cálculos utilizados nas técnicas espaciais do posicionamento geodésico se tornam mais simples quando se adota o GCRS. No caso das efemérides referenciadas no GCRS, a forma geométrica regular acarreta uma melhor solução das acelerações dos satélites, uma vez que não há aceleração no referencial (BLITZKOW et. al., 2011).

Ademais, com o intuito de comparar os modelos IAU2000A e IAU2000A/2006 para fins de posicionamento GNSS, as coordenadas do satélite do PRN (Pseudo-Random Number) 15 do GPS, para o dia 14 de julho de 2011, no referencial terrestre IGS08 (vinculado ao ITRS), foi transformada para o sistema GCRS. Para tanto, do Boletim B do IERS obteve-se os seguintes valores: $\mathrm{x}_{\mathrm{p}}=0,068788$ " e $\mathrm{y}_{\mathrm{p}}=0,444763$ ", e $D U T 1=-0,3 s$. As coordenadas no referencial terrestre é apresentado na Fig. 10. Vale destacar que a visualização das coordenadas apresentadas na Fig. 10 - bidimensional - caso projetada em três dimensões, teria um comportamento semelhante ao destacado na Fig. 8 tridimensional. As diferenças das coordenadas transformadas no referencial celeste com base na resolução IAU2000A e IAU2000A/2006 são mostradas na Fig. 11.

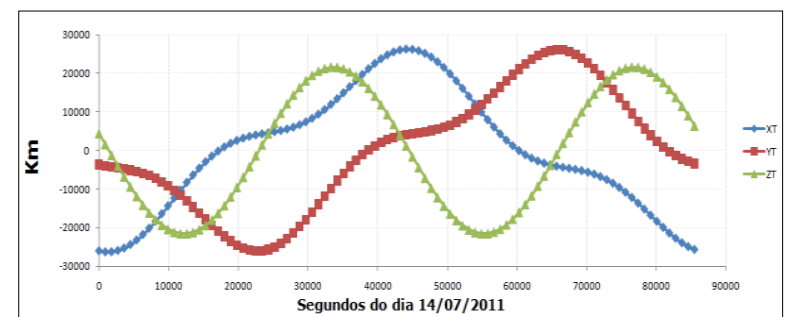

Figura 10 - Coordenadas do PRN 15 (GPS) no ITRS $(14 / 07 / 2011)$.

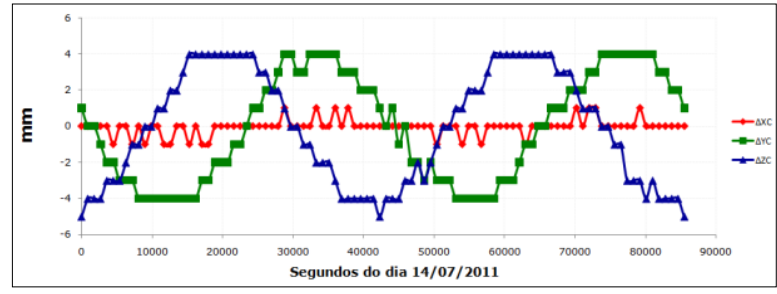

Figura 11 - Discrepância entre as coordenadas no sistema celeste IAU2000A e IAU2000A/2006 para o satélite PRN15 $(14 / 07 / 2011)$.

Nota-se pela Fig. 11, que a discrepância máxima é da ordem de $5 \mathrm{~mm}$ para a coordenada Z. Conclui-se que dependendo da aplicação as discrepâncias não são significativas entre os modelos IAU 2000 e IAU2000A/2006. No entanto, em aplicações de alta precisão, como por exemplo, no Posicionamento por Ponto Preciso (PPP) é recomendável considerar as resoluções em vigor, IAU2000A/2006.

\section{CONCLUSÕES}

Foram apresentados os padrões adotados pelo IERS para a definição do ICRS e do ITRS, bem como os conceitos utilizados na transformação do sistema celeste (GCRS) para o sistema terrestre (ITRS). Em tal transformação, o SOFA disponibiliza o código fonte em linguagem de programação $\mathrm{C}$, que possibilita a obtenção das matrizes de transformação entre os sistemas.

Atualmente, a função "iauC2t00a" permite obter tal matriz de transformação e possui os parâmetros $\mathrm{x}, \mathrm{y}$, UT1 do CIP no ITRS como parâmetros de entrada, sendo que estes são disponibilizados pelo IERS via os boletins A e B. A rotina implementada será útil para futuras investigações científicas, uma vez que, conforme os resultados apresentados, as efemérides referenciadas ao GCRS descrevem a movimentação dos satélites de forma regular, diferentemente ao movimento dos satélites no ITRS. As referências apresentadas no texto, bem como o acesso ao website http://www.iausofa.org/ permite aos leitores explorar outras possibilidades com mais detalhes.

\section{REFERÊNCIAS BIBLIOGRÁFICAS}

ALTAMIMI, Z.; COLLILIEUX (Ed.). Reference Frames for Applications in Geosciences. v. 138, ISBN 978-3-642-32998-2, 2013.

ALTAMIMI, Z.; COLLILIEUX, X.; MÉTIVIER, L. .ITRF2008: an improved solution of th e international terrestrial reference frame, Journal of Geodesy, v. 85, n. 8, pp. 457-473, 2011.

ALVES, D. B. M.; SOUZA, E. M.; MONICO, J. F. G. Movimento do Pólo Terrestre: Aspectos Físicos, Modelagem Matemática e Análises. Tend. Mat. Apl. Comput., 9, No. 1, 1-10, 2008.

BLITZKOW, D.; MATOS, A. C. O. C.; GUIMARÃES, G. N.; COSTA, S. M. A. O conceito atual dos 
referenciais usados em Geodésia. Revista Brasileira de Cartografia, n.63/5, p. 633-648, 2011.

BOCK, Y. Reference System. In: TEUNISSEN, P. J. G. and KLEUSBERG, A. (Eds.). GPS for Geodesy, Springer, 1996.

CAPITAINE, N.; SOFFEL, M. ON THE DEFINITION AND USE OF THE ECLIPTIC IN MODERN ASTRONOMY. Proceedings of the Journees Systemes de reference spatio-temporels, 2015.

CAPITAINE, N. Micro-arcsecond Celestial Reference Frames: definition and realization - Impact of the recent IAU Resolutions. Research in Astron. Astrophys. v. 12, n. 8, p. 1162-1184, 2012.

CAPITAINE, N., MATHEWS, P. M.; DEHANT , V.; WALLACE, P. T.; LAMBERT, S. B. On the IAU 2000/2006 precession-nutation and comparison with other models and VLBI observations. Celest. Mech. Dyn. Astr., v. 103, p.179-190, 2009.

CAPITAINE, N.; WALLACE, P.T.; CHAPRONT, J. Expressions for IAU 2000 precession quantities, Astron. Astrophys. v. 412, p.567-586, 2003.

CAPITAINE, N.; GUINOT, B.; McCARTHY, D. D. Definition of the Celestial Ephemeris Origin and of UT1 in the International Celestial Reference Frame Astronomy and Astrophysics. , v.355, p.398405, 2000.

IERS. IERS Conventions (2010). IERS Technical Note, No. 36, Frankfurt am Main, Germany: Verlag des Bundesamtes für Kartographie und Geodäsie, 2010.

McCARTHY, D. D.; LUZUM, B. J. Celest. Mech. Dyn. Astr. v. 85, n. 37, 2002.

McCARTHY, D.D. (Ed.). IERS Standards; IERS Technical Note 13. Observatoire de Paris, Paris, 1992.

MALKIN, et al. THE ICRF-3: STATUS, PLANS, AND PROGRESS ON THE NEXT GENERATION INTERNATIONAL CELESTIAL REFERENCE FRAME, 2014. Disponível em: < http://syrte.obspm.fr/jsr/journees2014/pdf/Malkin.pdf > Acesso em: 20 ago. 2015.

MATHEWS, P. M.; HERRING, T. A.; BUFFETT B. A. Modeling of nutation-precession: New nutation series for non-rigid Earth, and insights into the Earth's Interior. JGR, v. 107, B4, 10.1029/2001JB000390, 2002.

MONICO, J. F. G. Posicionamento pelo GNSS: Descrição, Fundamentos e Aplicações. 1 ed. São Paulo: Editora Unesp, 2008.
MONICO, J. F. G. FUNDAMENTOS MATEMÁTICOS ENVOLVIDOS NA REALIZAÇÃO DO ITRS. Bol. Ciênc. Geod., sec. Comunicações, Curitiba, v. 12, n. 2, p.337-351, jul-dez, 2006.

MONICO J. F. G. Transformação entre referenciais celeste e terrestre de acordo com a Resolução IAU 2000. In: Série em Ciências Geodésicas, vol. 4. Universidade Federal do Paraná. Curso de Pós Graduação em Ciências Geodésicas. Curitiba, v. 41, p. 31,2004 .

PLAG, H.-P.; GROSS, R.; ROTHACHER M. Global Geodetic Observing System for Geohazards and Global Change, Geosciences, 2009.

PLAG H.-P.; PEARLMAN M. (Ed.), Global Geodetic Observing System. Meeting the Requirements of a Global Society on a Changing Planet in 2020. Springer Verlag, 2009.

SEEBER, G., Satellite Geodesy: foundations, methods and applications. Berlin, NewYork: Walter de Gruyter, 2003. 